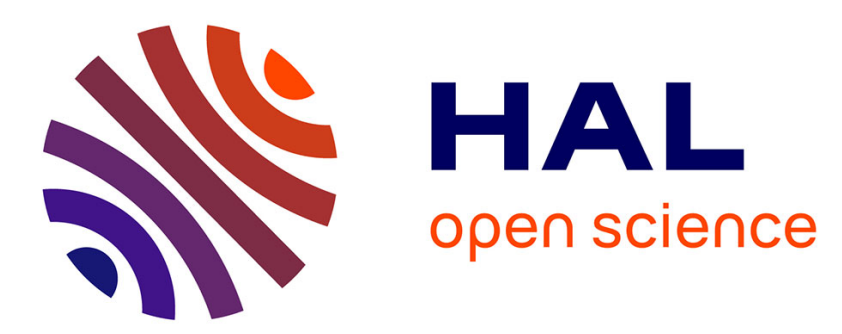

\title{
Stability analysis and robustness results for a nonlinear system with distributed delays describing hematopoiesis
}

Walid Djema, Frédéric Mazenc, Catherine Bonnet

\section{To cite this version:}

Walid Djema, Frédéric Mazenc, Catherine Bonnet. Stability analysis and robustness results for a nonlinear system with distributed delays describing hematopoiesis. Systems and Control Letters, 2017, 102, pp.93 - 101. 10.1016/j.sysconle.2017.01.007 . hal-01627125

\section{HAL Id: hal-01627125 \\ https://hal.inria.fr/hal-01627125}

Submitted on 22 Jan 2018

HAL is a multi-disciplinary open access archive for the deposit and dissemination of scientific research documents, whether they are published or not. The documents may come from teaching and research institutions in France or abroad, or from public or private research centers.
L'archive ouverte pluridisciplinaire HAL, est destinée au dépôt et à la diffusion de documents scientifiques de niveau recherche, publiés ou non, émanant des établissements d'enseignement et de recherche français ou étrangers, des laboratoires publics ou privés. 


\title{
Stability Analysis and Robustness Results for a Nonlinear System with Distributed Delays Describing Hematopoiesis. tर $^{\overrightarrow{2}}$
}

\author{
Walid Djema ${ }^{\mathrm{a}}$, Frédéric Mazenc ${ }^{\mathrm{a}}$, Catherine Bonnet ${ }^{\mathrm{a}}$ \\ ${ }^{a}$ Inria, Université Paris-Saclay, L2S-CentraleSupélec, 3 rue Joliot Curie, 91192, \\ Gif-sur-Yvette, France. walid.djema@inria.fr \\ frederic.mazenc@12s. centralesupelec.fr catherine.bonnet@inria.fr
}

\begin{abstract}
A nonlinear system with distributed delays describing cell dynamics in hematopoiesis is analyzed -in the time-domain- via a construction of suitable LyapunovKrasovskii functionals (LKFs). Two interesting biological situations lead us to re-investigate the stability properties of two meaningful steady states: the 0 -equilibrium for unhealthy hematopoiesis and the positive equilibrium for the healthy case. Biologically, convergence to the 0-equilibrium means the extinction of all the generations of blood cells while the positive equilibrium reflects the normal process where blood cells survive. Their analyses are slightly different in the sense that we take advantage of positivity of the system to construct linear functionals to analyze the 0-equilibrium, while we use some quadratic functionals to investigate the stability properties of the positive equilibrium. For both equilibria, we establish the exponential stability of solutions and we provide an estimate of their rates of convergence. Moreover, a robustness analysis is performed when the model is subject to some nonvanishing perturbations. Numerical examples are provided.
\end{abstract}

Keywords: Delay, Positive system, Lyapunov, Stability, Biological model.

\section{Introduction}

With the ultimate goal of determining a model describing cell dynamics in acute myeloid leukemia, which will be of use for the optimization of polychemotherapies, we start here with a model describing the process of fabrication 5 of blood which was studied in [1] and revisited by input-output methods in [16]. Using an alternative approach, our aim here is to deepen the analysis as well as to solve some open issues which are of importance in practice.

Through the process of hematopoiesis, the Hematopoeitic Stem Cells (HSCs) develop into red blood cells, white blood cells, platelets and all other blood cells.

Supported by ALMA3 project on the 'Analysis of Acute Myeloid Leukemia', through DIGITEO, Paris-Saclay. 
HSCs are immature unspecialized cells able to produce cells with the same maturity level and to differentiate into specialized cells. This is a simplified development scheme, which does not take into account other cell fates -increasingly highlighted in recent years- such as cell dedifferentiation [4. In fact, the complex cascade of signals regulating hematopoiesis is not currently clearly identi-

15 fied. Therefore, the importance of this biological process has motivated many theoretical and experimental works that focus on the earliest generations of immature cells since they play a critical role in blood formation, and because they are the source of several hematological disorders. The long list of works devoted to blood cells dynamics includes [1, [2], 3], [13, [16, [17, 12], 18, and [8].

20 Acute Myelogenous Leukemia (AML) is a serious type of cancer, which is characterized by an overproduction of abnormal myeloblasts, simultaneously with an inability to develop further into mature white blood cells (a blockade in the maturation process). Due to their overproliferation, blasts invade the bone marrow and even - sometimes - the blood circulation (Figure 1 $\mathrm{ra}$ ), which prevents adequate production of mature healthy blood cells. Since we want to emphasize on AML, we consider that the model that we focus on describes the development hierarchy leading to white cell production in the myeloid lineage.

Relying on several essential contributions by Mackey and his colleagues ([12], [18, 13, to name but a few), Adimy et al. introduced and analyzed in [1] a 30 nonlinear system with distributed delays to model cell dynamics in several maturity stages. This is the model we study here, considering that it describes a cancer state when some of its biological parameters are abnormal (i.e. being different from healthy parameters, or becoming time-varying to model the effect of appropriate infused drugs) and it reflects a healthy situation when all its 35 parameters are normal. Using a Lyapunov technique we improve some existing results in two different contexts: i) we provide theoretical conditions to eradicate cancer cells in what we assume to be a basic unhealthy situation, and, ii) we ensure the survival of healthy cells in normal hematopoiesis. A key point that we emphasize here is that the Lyapunov direct method offers strong tools to 40 study exponential convergence of solutions, estimates on their decay rates (for both steady states), as well as estimating the basin of attraction of the positive equilibrium point and this, in our opinion, improves the way to study the phenomenon of hematopoiesis (see the concluding remarks in [16]). On the other hand, the search for a suitable Lyapunov functional is generally quite difficult, 45 since no systematic methods apply [14, 10, and that is the challenging problem that we are dealing with in this contribution.

The paper is organized as follows. In Section 2 we briefly present the model of interest. Section 3 is devoted to the study of the 0 -equilibrium of the system. We establish global exponential stability even when some parameters are time50 varying, then we perform a robustness analysis. The strictly positive equilibrium $X^{e}$ of the nominal system is discussed in Section 4. An estimate of its basin of attraction is proposed via a construction of a novel Lyapunov functional, that also allows us to perform a robustness analysis of the perturbed system. 


\section{Description of the model and known results}

We revisit from [1] the model described in Figure 1-b, where for all $i \in I_{n}=$ $\{1, \ldots, n\}, n \geq 1, x_{i}$ denotes the total density of resting cells of generation $i$. A resting cell is a cell that is not actively in the process of dividing. The reintroduction function from resting into proliferating subpopulation of the $i$-th generation is denoted $\beta_{i}(\cdot)$. Proliferating cells can divide between the moment they enter the proliferating phase and a maximal age $\tau_{i}>0$, while the apoptosis rate, $\gamma_{i}$, represents the death rate of proliferating cells of the $i$-th generation. At each division, a proportion $K_{i}$ of dividing cells goes to the next resting stage of the development hierarchy of interest, while the other part $\left(L_{i}=1-K_{i}\right)$ stays at the same level $i$ (self-renewing process), with the convention that $K_{0}=0$. The constant $\delta_{i}$ covers both the death rate of the resting cells of the $i$-th generation, together with their differentiation into lineages that we do not focus on.



Figure 1: (a) Blast cells are not typically found in the circulating blood of healthy individuals. The picture is from the National Cancer Institute. (b) Schematic representation of the earliest stages in the myeloid lineage [1].

Finally, the dynamical system equation is in the form:

$$
\begin{aligned}
\dot{x}_{i}(t)= & -\delta_{i} x_{i}(t)-w_{i}\left(x_{i}(t)\right)+2 L_{i} \int_{0}^{\tau_{i}} g_{i}(a) w_{i}\left(x_{i}(t-a)\right) d a \\
& +2 K_{i-1} \int_{0}^{\tau_{i-1}} g_{i-1}(a) w_{i-1}\left(x_{i-1}(t-a)\right) d a+\epsilon_{i}(t),
\end{aligned}
$$

for each compartment $i \in I_{n}$, and $t \geq 0$, with $w_{i}\left(x_{i}\right)=\beta_{i}\left(x_{i}\right) x_{i}, g_{i}(a)=$ $e^{-\gamma_{i} a} f_{i}(a)$, where the $f_{i}$ s are $\mathcal{C}^{1}$ functions representing the cell division probability densities, such that $f_{i}(a) \geq 0$ for all $a \in\left[0, \tau_{i}\right]$, and $\int_{0}^{\tau_{i}} f_{i}(a) d a=1$, since it is assumed in [1] that the mitosis occurs before the age-limit $\tau_{i}$. Moreover, biological facts induce that the parameters $\delta_{i}, L_{i}, K_{i}, \tau_{i}$ and $\gamma_{i}$ are positive for all $i \in I_{n}$, with $K_{0}=0$ and $K_{i} \in(0,1)$ for all $i \in I_{n}$. The functions $\beta_{i}(\cdot)$ are assumed to be differentiable and decreasing functions such that $\lim _{a \rightarrow+\infty} \beta_{i}(a)=0$. For a later use, we introduce the following parameters:

$$
C_{i}=\int_{0}^{\tau_{i}} g_{i}(l) d l, \quad \text { and }, \quad \alpha_{i}=2 L_{i} C_{i}-1,
$$

where $\alpha_{i}$ is assumed to be strictly positive, for all $i \in I_{n}$ (see [16], Assumption 2). We will perform a robustness analysis of the model (1) under nonvanishing 
perturbation terms $\epsilon_{i}(t) \in\left[0, \bar{\epsilon}_{i}\right]$, where $\bar{\epsilon}_{i}>0$, for all $i \in I_{n}$ and $t \geq 0$.

70 It is well-known that disturbances are in general due to the lack of accuracy when modeling the laws governing complex living organisms. More precisely, in the model that we study, uncertainty comes from the biological parameters and functions (e.g. the nonlinearity $\beta_{i}$, introduced in [12]), and from more complex phenomena which are difficult to model. In particular, the ability of 75 differentiated cells to undergo lineage reversion (including dedifferentiation - the mechanism whereby differentiated cells regress to a less mature state 4] - and transdifferentiation from different types of cells and hierarchies) is not covered by the model illustrated in Figure 1 $\mathrm{b}$. A basic representation of cells plasticity features is achieved by considering dedifferentiation and transdifferentiation as

so perturbation inputs. In fact, it can be proven that nonvanishing perturbations arise from cell plasticity, and uncertain re-introduction functions $\beta_{i}$, leading to system (1) with $\epsilon_{i}(t) \in\left(0, \bar{\epsilon}_{i}\right]$, for all $t \geq 0$.

\section{Notation and definitions:}

Throughout the paper, we analyze the stability of the model described by (1),

85 where for all $i \in I_{n}=\{1, \ldots, n\}, x_{i}(t) \in \mathbb{R}^{n}$. The state of the system (1) at a time instant $t$ is defined as the restriction of each component $x_{i}(t)$ of the solution $x(t)=\left(x_{1}(t), \ldots, x_{n}(t)\right)$, on the segment $\left[t-\tau_{i}, t\right]$, for all $i \in I_{n}$. We let $x=\left(x_{1}, \ldots, x_{n}\right)$ and $\mathcal{C}_{\text {in }}=\mathcal{C}\left(\left[-\tau_{i}, 0\right], \mathbb{R}\right)$ denote the space of all continuous $\mathbb{R}$-valued functions defined on a given interval $\left[-\tau_{i}, 0\right]$, for all $i \in I_{n}$, and for all 9o $t \geq 0$, the function $x_{i t}$ is defined by $x_{i t}(m)=x_{i}(t+m)$ for all $m \in\left[-\tau_{i}, 0\right]$.

Finally, we notice that negative steady states are excluded from this study, as well as equilibria belonging to the boundaries of the positive orthant, except the origin, because of their biological irrelevance. We focus on two meaningful steady states: the 0 -equilibrium denoted by $X^{0}=(0, \ldots, 0)$ and the strictly positive equilibrium point denoted by $X^{e}=\left(x_{1}^{e}, \ldots, x_{n}^{e}\right), x_{i}^{e}>0$, for all $i \in I_{n}$.

We recall two simple but useful results (see [1] and [5]).

Proposition 1. The solutions of the system (1) with positive initial conditions are positive.

100 Proof. Since the nonvanishing perturbations $\epsilon_{i}(t)$, for all $i \in I_{n}$, satisfy $\epsilon_{i}(t) \geq 0$, for all $t \geq 0$, the proof that the positive orthant is forward invariant is similar to the one proposed in [5] for the nominal system.

Concerning steady states for the nominal system (1), we notice that $X^{0}$ always exists. Next, without proof (available in [1] and [5]), we recall the necessary and sufficient condition of the existence of $X^{e}$ of the nominal system (1):

Proposition 2. The nominal system (1) admits a positive equilibrium point $X^{e}=\left(x_{1}^{e}, \ldots, x_{n}^{e}\right)$ if and only if:

$$
\beta_{1}(0)>\frac{\delta_{1}}{\alpha_{1}} .
$$

Throughout Section 4, we will assume that the condition (3) is satisfied and we will analyze the stability properties of $X^{e}$ using a new approach. 


\section{Stability analysis of the trivial steady state}

\subsection{Global exponential stability of the nominal system}

We start by establishing global exponential stability of the origin $X^{0}$. As a corollary of this result, we prove exponential stability of $X^{0}$ when some biological parameters are uncertain or time-varying.

Theorem 1. The nominal system (1) admits the origin, $X^{0}$, as a globally exponentially stable equilibrium point if for all $i \in I_{n}$, the inequalities

$$
s_{i}:=\delta_{i}-\left(2 C_{i} L_{i}-1\right) \beta_{i}(0)>0,
$$

are satisfied. If

$$
s_{1}:=\delta_{1}-\left(2 C_{1} L_{1}-1\right) \beta_{1}(0)<0,
$$

then no positive solution converges to $X^{0}$.

Remark 1. i) We can readily check that if (4) is satisfied, then zero is the unique equilibrium of the nominal system (1). ii) Using a frequency domain approach, it was proved in [1] that if (5) is satisfied then the system is unstable, and that the conditions (4) guarantee local asymptotic stability of the origin. In [3], slightly more restrictive conditions than (4) (due to the fact that $L_{i}<1$, for all $i \in I_{n}$ ) were given to ensure global asymptotic stability of the origin. In [5], we proved global asymptotic stability of $X^{0}$ under conditions (4). In Theorem 1 of the present paper, we extend the result of [5] to establish global exponential stability under conditions (4). iii) Even if the analytic expression of the Lyapunov functional that we will introduce in this section will be slightly different from the one used in [5], they share the common feature of being unusual since they are approximated at the origin by linear functions.

Proof. First, let us pick a family of positive constants $\rho_{i}$, to be selected later, and define for all $i \in I_{n}$, the functionals

$$
v_{i}\left(x_{i t}\right)=\int_{t-\tau_{i}}^{t} \int_{m}^{t} e^{-\rho_{i}\left(t-m-\tau_{i}\right)} g_{i}\left(m+\tau_{i}-a\right) w_{i}\left(x_{i}(a)\right) d a d m .
$$

For all $i \in I_{n}$, the derivative of the functional (6) along the trajectories of the nominal system (1) satisfies

$$
\begin{aligned}
\dot{v}_{i}(t) & =-\rho_{i} v_{i}\left(x_{i t}\right)-\int_{t-\tau_{i}}^{t} g_{i}(t-a) w_{i}\left(x_{i}(a)\right) d a+w_{i}\left(x_{i}(t)\right) \int_{0}^{\tau_{i}} e^{\rho_{i} a} g_{i}(a) d a \\
& \leq-\rho_{i} v_{i}\left(x_{i t}\right)-\int_{t-\tau_{i}}^{t} g_{i}(t-a) w_{i}\left(x_{i}(a)\right) d a+w_{i}\left(x_{i}(t)\right) e^{\rho_{i} \tau_{i}} C_{i},
\end{aligned}
$$

where the last inequality is a consequence of (2). Let us introduce the following functional for the first compartment of hematopoietic stem cells:

$$
\mathcal{V}_{1}\left(x_{1 t}\right)=x_{1}(t)+2 L_{1} v_{1}\left(x_{1 t}\right) .
$$


Its derivative along the trajectories of the nominal system (1) satisfies

$$
\dot{\mathcal{V}}_{1}(t) \leq-\delta_{1} x_{1}(t)-2 L_{1} \rho_{1} v_{1}\left(x_{1 t}\right)-\left[1-2 L_{1} e^{\rho_{1} \tau_{1}} C_{1}\right] w_{1}\left(x_{1}(t)\right) .
$$

Since $\alpha_{1}>0$, we conclude that for all $\rho_{1}>0$, the inequality $2 L_{1} e^{\rho_{1} \tau_{1}} C_{1}-1>0$ is satisfied. Therefore, using $w_{1}\left(x_{1}(t)\right) \leq \beta_{1}(0) x_{1}(t)$, it follows from (8) that:

$$
\dot{\mathcal{V}}_{1}(t) \leq-\left[\delta_{1}-\left(2 L_{1} e^{\rho_{1} \tau_{1}} C_{1}-1\right) \beta_{1}(0)\right] x_{1}(t)-2 L_{1} \rho_{1} v_{1}\left(x_{1 t}\right) .
$$

Now, if (4) is satisfied, we choose $\rho_{1}=\frac{1}{2 \tau_{1}} \ln \left(\frac{\delta_{1}+\beta_{1}(0)+2 L_{1} C_{1} \beta_{1}(0)}{4 L_{1} C_{1} \beta_{1}(0)}\right)$. Then we obtain $\delta_{1}-\left(2 L_{1} e^{\rho_{1} \tau_{1}} C_{1}-1\right) \beta_{1}(0) \geq \frac{s_{1}}{2}$. It follows that the inequality (9) gives $\dot{\mathcal{V}}_{1}(t) \leq-\frac{s_{1}}{2} x_{1}(t)-2 L_{1} \rho_{1} v_{1}\left(x_{1 t}\right)$, and from the definition of $\mathcal{V}_{1}$, we get

$$
\dot{\mathcal{V}}_{1}(t) \leq-\tilde{s}_{1} \mathcal{V}_{1}\left(x_{1 t}\right)-\frac{s_{1}}{4} x_{1}(t)
$$

with $\tilde{s}_{1}=\min \left\{\frac{s_{1}}{4}, \rho_{1}\right\}$. Consequently, the origin of the subsystem $i=1$ is globally exponentially stable. Next, in order to extend the result to the overall system, we introduce the following functional which takes into account the cells dynamics of the first and the second generations of immature cells:

$$
\mathcal{V}_{2}\left(x_{2 t}, x_{1 t}\right)=x_{2}(t)+2 L_{2} v_{2}\left(x_{2 t}\right)+2 K_{1} v_{1}\left(x_{1 t}\right)+\frac{8 K_{1} \beta_{1}(0) e^{\rho_{1} \tau_{1}} C_{1}}{s_{1}} \mathcal{V}_{1}\left(x_{1 t}\right)
$$

Using (10), we prove that the derivative of $\mathcal{V}_{2}$ along the trajectories of the nominal system (1) satisfies

$$
\begin{aligned}
\dot{\mathcal{V}}_{2}(t) \leq & -\delta_{2} x_{2}(t)-\left(1-2 L_{2} e^{\rho_{2} \tau_{2}} C_{2}\right) w_{2}\left(x_{2}(t)\right)-2 L_{2} \rho_{2} v_{2}\left(x_{2 t}\right) \\
& -2 K_{1} \rho_{1} v_{1}\left(x_{1 t}\right)-\frac{8 K_{1} \beta_{1}(0) e^{\rho_{1} \tau_{1}} C_{1} \tilde{s}_{1}}{s_{1}} \mathcal{V}_{1}\left(x_{1 t}\right) \\
& -2 K_{1} e^{\rho_{1} \tau_{1}} C_{1}\left[\beta_{1}(0)-\beta_{1}\left(x_{1}(t)\right)\right] x_{1}(t) .
\end{aligned}
$$

Using the assumption $\alpha_{2}>0$, together with the fact that the function $\beta_{2}$ is strictly decreasing, it follows that,

$$
\begin{aligned}
\dot{\mathcal{V}}_{2}(t) \leq & -\left[\delta_{2}-\left(2 L_{2} e^{\rho_{2} \tau_{2}} C_{2}-1\right) \beta_{2}(0)\right] x_{2}(t)-2 L_{2} \rho_{2} v_{2}\left(x_{2 t}\right) \\
& -2 K_{1} \rho_{1} v_{1}\left(x_{1 t}\right)-\frac{8 K_{1} \beta_{1}(0) e^{\rho_{1} \tau_{1}} C_{1} \tilde{s}_{1}}{s_{1}} \mathcal{V}_{1}\left(x_{1 t}\right) .
\end{aligned}
$$

When the conditions (4) are satisfied, we select $\rho_{2}>0$ (similarly to $\rho_{1}$ ), such that the inequality $\delta_{2}-\left(2 L_{2} e^{\rho_{2} \tau_{2}} C_{2}-1\right) \beta_{2}(0) \geq \frac{s_{2}}{2}$, is satisfied. It follows from (12) that there exists a strictly positive constant $\tilde{s}_{2}$, such that

$$
\dot{\mathcal{V}}_{2}(t) \leq-\tilde{s}_{2} \mathcal{V}_{2}\left(x_{1 t}, x_{2 t}\right)-\frac{s_{2}}{4} x_{2}(t),
$$

is satisfied. Next, by induction, we easily check that there exist a positive constant $\tilde{s}_{n}$ and a family of strictly positive weighting constants $\lambda_{i}$ and $\tilde{\lambda}_{i}$, such 
that the derivative of the functional $\mathcal{V}\left(x_{t}\right)=\sum_{i=1}^{n}\left[\lambda_{i} x_{i}(t)+\tilde{\lambda}_{i} v_{i}\left(x_{i t}\right)\right]$, which is taking into account all the $n$ generations of immature blood cells, along the trajectories of the nominal system (1), satisfies

$$
\dot{\mathcal{V}}(t) \leq-\tilde{s}_{n} \mathcal{V}\left(x_{t}\right)
$$

From the inequality (14) and the properties of the functional $\mathcal{V}$, we conclude that, if the conditions (4) are satisfied, the origin of the nominal model (1) is globally exponentially stable.

In order to complete the proof, we consider the case where the inequality (5) is satisfied and we show that no positive solution converges to $X^{0}$. As in [5], we prove this result by contradiction, i.e. we assume that a positive solution $x(t)$ converges to $X^{0}$. Now, we select $\rho_{1}=0$ and we observe that the derivative of the functional $\mathcal{V}_{1}$, introduced in (7), is given by

$$
\dot{\mathcal{V}}_{1}(t)=\left[-\delta_{1}+\alpha_{1} \beta_{1}\left(x_{1}(t)\right)\right] x_{1}(t)
$$

When (5) is verified, using the facts that the function $\beta_{1}$ is decreasing and $x_{1}(t)$ converges to zero, we deduce that there exists $t_{r}>0$ such that, for all $t \geq t_{r}$, $-\delta_{1}+\alpha_{1} \beta_{1}\left(x_{1}(t)\right) \geq \frac{-\delta_{1}+\alpha_{1} \beta_{1}(0)}{2}$. It follows from 15 that, for all $t \geq t_{r}$,

$$
\dot{\mathcal{V}}_{1}(t) \geq \frac{-\delta_{1}+\alpha_{1} \beta_{1}(0)}{2} x_{1}(t)
$$

From (5), and the positivity of the solutions, it follows that for all $t \geq t_{r}$, $\dot{\mathcal{V}}_{1}(t)>0$. Consequently, we deduce that, for all $t \geq t_{r}, \mathcal{V}_{1}\left(x_{1 t}\right) \geq \mathcal{V}_{1}\left(x_{1 t_{r}}\right)>0$. It follows that $\mathcal{V}_{1}\left(x_{1 t}\right)$ does not converge to zero. On the other hand, $\mathcal{V}_{1}\left(x_{1 t}\right)$ converges to zero because $x_{1}(t)$ converges to $X^{0}$. This yields a contradiction.

Example 1. A possible selection of the cell division probability densities, which was considered in [17] and [16], is given by $f_{i}(a)=\frac{m_{i}}{e^{m_{i} \tau_{i}}-1} e^{m_{i} a}$, with $m_{i}>0$, for all $i \in I_{n}$. Let us consider the following biological functions and parameters:

\begin{tabular}{|c||c||c||c||c||c||c|}
\hline & $\beta_{i}\left(x_{i}\right)$ & $f_{i}(a)$ & $\delta_{i}$ & $L_{i}$ & $\tau_{i}$ & $\gamma_{i}$ \\
\hline$i=1$ & $\frac{1.22}{1+x_{1}^{2}}$ & $\frac{5 e^{5 a}}{e^{5 \tau} 1-1}$ & 0.9 & 0.85 & 1.2 & 0.22 \\
\hline$i=2$ & $\frac{1.33}{1+4 x_{2}^{4}}$ & $\frac{7 e^{7 a}}{e^{7 \tau}-1}$ & 0.96 & 0.8 & 1.3 & 0.33 \\
\hline
\end{tabular}

The form given to $\beta_{i}$ [12] normalizes the values taken by the total density $x_{i}$. Simple calculations give: $\left(2 L_{1} C_{1}-1\right) \beta_{1}(0)=0.4448,\left(2 L_{2} C_{2}-1\right) \beta_{2}(0)=$ 0.4392. Therefore, according to Proposition 2, the positive equilibrium of system (1) does not exist. Moreover, according to Theorem 1, the origin $X^{0}=(0,0)$ of system (1) is globally exponentially stable, as shown in Figure 2

\subsection{Global exponential stability under time-varying differentiation rates}

Convergence to $X^{0}$ means the eradication of all the immature blood cells. This case may be suitable when the model is assumed to describe the dynamics of unhealthy cells. Recall that one of the characteristics of leukemia is the blockade in the differentiation process, which becomes also a target for the drugs used in 
treatments. From a theoretical point of view, it is interesting to consider the case where differentiation and self-renewing rates are uncertain or time varying.

In this part, we extend the result of Theorem 1 to the nominal model that describes the immature cell dynamics under time-varying differentiation rates, $K_{i}(t)$ for all $t \geq 0$, and $i \in I_{n}$, and which is given by

$$
\begin{aligned}
\dot{x}_{i}(t)= & 2 K_{i-1}(t) \int_{0}^{\tau_{i-1}} g_{i-1}(a) w_{i-1}\left(x_{i-1}(t-a)\right) d a \\
& +2 L_{i}(t) \int_{0}^{\tau_{i}} g_{i}(a) w_{i}\left(x_{i}(t-a)\right) d a-\delta_{i} x_{i}(t)-w_{i}\left(x_{i}(t)\right),
\end{aligned}
$$

where $K_{i}(t)+L_{i}(t)=1$ and $L_{i}(t) \in\left[L_{i \min }, L_{i \max }\right] \subset(0,1)$. We recall that, by

convention, $K_{0}(t)=0$, for all $t \geq 0$, and we assume that $K_{i}(\cdot), L_{i}(\cdot)$ are of class $C^{0}$, for all $i \in I_{n}$. Based on Theorem 1, we prove the following result:

Corollary 1. The conditions

$$
\bar{s}_{i}=\delta_{i}-\left(2 L_{i \max } C_{i}-1\right) \beta_{i}(0)>0, \quad \forall i \in I_{n},
$$

ensure that the origin of the system (17) is globally exponentially stable.

Proof. We give some indications for the proof, which is slightly different from the one of Theorem 1 . Here we consider $L_{1 \text { max }}$ instead of $L_{1}$ in the definition of the functional $\mathcal{V}_{1}\left(x_{1 t}\right)$ introduced in (7), and, similarly, we consider $L_{2 \max }$, $K_{1 \max }=1-L_{1 \min }$ and $\bar{s}_{1}$, instead of $L_{2}, K_{1}$, and $s_{1}$, respectively, in the definition of the functional $\mathcal{V}_{2}\left(x_{2 t}, x_{1 t}\right)$.

Example 2. Let us consider $n=2$ and for all $t \geq 0, L_{1}(t)=\frac{1}{2}(1+0.97 \cos (25 t))$ and $L_{2}(t)=\frac{1}{2}(1+0.97 \sin (15 t))$. Sine function sounds reasonable to model the variation in differentiation rates since drugs are - usually - infused quasiperiodically. Nevertheless, many other time-varying functions may be used instead of sine ones. Let us assume that:

\begin{tabular}{|c||c||c||c||c||c|}
\hline & $\beta_{i}\left(x_{i}\right)$ & $f_{i}(a)$ & $\delta_{i}$ & $\tau_{i}$ & $\gamma_{i}$ \\
\hline$i=1$ & $\frac{2.87}{1+x_{1}^{2}}$ & $\frac{e^{a}}{e^{\tau_{1}}-1}$ & 0.973 & 0.8 & 0.9 \\
\hline$i=2$ & $\frac{2.7}{1+x_{2}^{4}}$ & $\frac{e^{a}}{e^{\tau}-1}$ & 0.965 & 0.7 & 0.97 \\
\hline
\end{tabular}

Elementary calculations give: $\bar{s}_{1}=0.0592$, and, $\bar{s}_{2}=0.0099$.

According to Corollary $1, X^{0}=(0,0)$, which is the unique equilibrium point of (17), is globally exponentially stable. Figure 3 illustrates the trajectories $x_{1}$ and $x_{2}$ for the the parameters and biological functions of Example 2.

Remark 2. At this juncture, we emphasize that Theorem 1 and Corollary 1 complement the results of [1], [3], and [5], by establishing global exponential stability instead of asymptotic stability and by extending the result to cover the case of time-varying differentiating and self-renewing rates. Let us briefly comment these results in the AML case, in which we expect a blockade of differentiation, i.e. $K_{i}$ decreases in early maturity stages. Not surprisingly, the conditions (18) suggest that therapeutic strategies to eradicate cells must be oriented towards increasing the death rates $\gamma_{i}$ (recall that increasing the apoptosis rate $\gamma_{i}$ decreases 


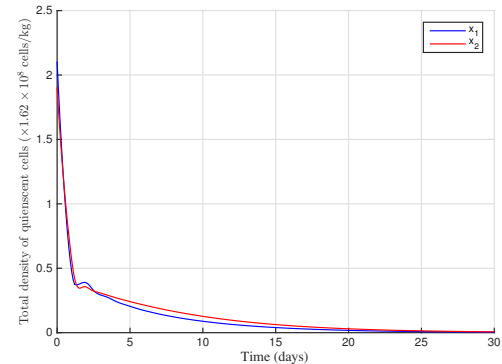

Figure 2: Trajectories of Example 1.

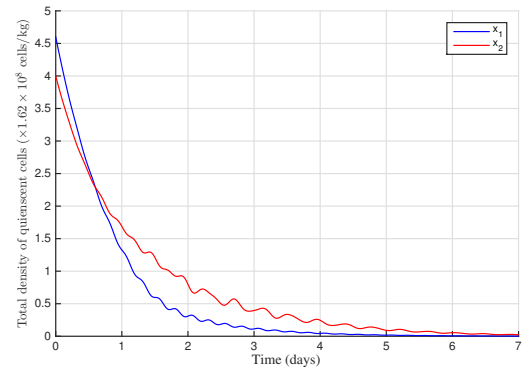

Figure 3: Trajectories of Example 2.

$C_{i}$ ), and $\delta_{i}$, and also towards decreasing $L_{\text {imax }}$ (i.e. increasing differentiation). Although very partial results for particular cases of AML (with myelodysplastic syndrome, $M D S)$, and on cell cultures only, have been obtained using tyrosine kinase inhibitors (TKIs, in particular dasatinb [11]) in stimulating differentiation, the only clinically efficient case of redifferentiation therapy known until recently was by using all-trans retinoic acid (ATRA) and arsenic tri-oxide in acute promyelocytic leukemia (APL). However, this therapeutic track has lately been relaunched by establishing that inhibition of Dihydroorotate Dehydrogenase $(D H O D H)$ is efficient in releasing cells from differentiation arrest [15]. Finally, increasing apoptosis may be achieved classically by using cytosine arabinoside.

\subsection{Robustness analysis of the trivial steady state}

In this section, we use the strict functionals $\mathcal{V}_{i}$, introduced in Theorem 1 , to perform a robustness analysis in the case of nonvanishing perturbations $\epsilon_{i}(t) \in$ $\left(0, \bar{\epsilon}_{i}\right]$, for all $i \in I_{n}, t \geq 0$. Let us observe that the derivative of the functional $\mathcal{V}_{1}$, defined in (7), along the trajectories of the perturbed system (1), satisfies,

$$
\dot{\mathcal{V}}_{1}(t) \leq-\tilde{s}_{1} \mathcal{V}_{1}\left(x_{1 t}\right)-\frac{s_{1}}{4} x_{1}(t)+\bar{\epsilon}_{1} .
$$

We consider any constant $\theta \in(0,1)$ and we define the family of sets:

$$
\mathcal{T}_{i \bar{\epsilon}_{i}}=\left\{\varphi \in \mathcal{C}\left(\left[-\tau_{i}, 0\right], \mathbb{R}^{+}\right), \quad \mathcal{V}_{i}(\varphi) \leq \frac{\bar{\epsilon}_{i}}{\theta \tilde{s}_{i}}\right\}
$$

Notice for later use that the sets $\mathcal{T}_{i \bar{\epsilon}_{i}}$ are the smallest possible for $\theta$ close to 1. Clearly, if $x_{1 t} \notin \mathcal{T}_{1 \bar{\epsilon}_{1}}$, then (19) gives $\dot{\mathcal{V}}_{1}(t) \leq-(1-\theta) \tilde{\mathcal{S}} \mathcal{V}_{1}\left(x_{1 t}\right)-\frac{s_{1}}{4} x_{1}(t)$. Therefore, the state $x_{1 t}$ converges exponentially to the set $\mathcal{T}_{1 \bar{\epsilon}_{1}}$. However, a refined result can be provided, in the sense that we can determine smaller positive invariant sets than the family $\mathcal{T}_{i \bar{\epsilon}_{i}}$. For that, let us introduce the functional,

$$
\mathcal{A}_{1}\left(x_{1 t}\right)=\mathcal{V}_{1}\left(x_{1 t}\right)-\psi_{1}^{\dagger} x_{1}(t) .
$$

It is worth mentioning that the functional $\mathcal{A}_{1}$ is positive on the positive orthant for $\psi_{1}^{\dagger}=\frac{s_{1}}{8\left(\delta_{1}+\beta_{1}(0)\right)}<1$, where $s_{1}$ is the constant defined in (4). Using the 
expression of $\psi_{1}^{\dagger}$, we can check that the derivative of $\mathcal{A}_{1}$, along the trajectories of the perturbed system (1), satisfies:

$$
\dot{\mathcal{A}}_{1}(t) \leq-\tilde{s}_{1} \mathcal{V}_{1}\left(x_{1 t}\right)-\frac{s_{1}}{8} x_{1}(t)-2 \psi_{1}^{\dagger} L_{1} \int_{t-\tau_{1}}^{t} g_{1}(t-a) w_{1}\left(x_{1}(a)\right) d a+\left(1-\psi_{1}^{\dagger}\right) \bar{\epsilon}_{1}
$$

Now, if we define the family of sets:

$$
\tilde{\mathcal{T}}_{i \bar{\epsilon}_{i}}=\left\{\varphi \in \mathcal{C}\left(\left[-\tau_{i}, 0\right], \mathbb{R}^{+}\right), \mathcal{V}_{i}(\varphi)+\frac{2 \psi_{i}^{\dagger} L_{i}}{\tilde{s}_{i} \theta} \int_{-\tau_{i}}^{0} g_{i}(a) w_{i}(\varphi) d a \leq \frac{\left(1-\psi_{i}^{\dagger}\right) \bar{\epsilon}_{i}}{\theta \tilde{s}_{i}}\right\}
$$

where $0<\psi_{i}^{\dagger}<1$, for all $i \in I_{n}$. Observe that $\tilde{\mathcal{T}}_{i \bar{\epsilon}_{i}} \subset \mathcal{T}_{i \bar{\epsilon}_{i}}$, for all $\psi_{i}>0$. Now, notice that for all $x_{1 t} \notin \tilde{\mathcal{T}}_{1 \bar{\epsilon}_{1}}$, the derivative of the functional $\mathcal{A}_{1}$ satisfies,

$$
\dot{\mathcal{A}}_{1}(t) \leq-\tilde{s}_{1}(1-\theta) \mathcal{V}_{1}\left(x_{1 t}\right)-\frac{s_{1}}{8} x_{1}(t) \leq-\tilde{s}_{1 \theta} \mathcal{A}_{1}\left(x_{1 t}\right)-\frac{s_{1}+\psi_{1}^{\dagger} \theta}{8} x_{1}(t),
$$

where $\tilde{s}_{1 \theta}=\min \left\{\tilde{s}_{1}(1-\theta), \theta / 8\right\}>0$, for all $\theta \in(0,1)$. Therefore, from the definition of the functional $\mathcal{A}_{1}$, we conclude that the state $x_{1 t}$ converges exponentially to $\tilde{\mathcal{T}}_{1 \bar{\epsilon}_{1}}$, and the decay rate of the trajectory $x_{1}(t)$ is smaller than, or equal to, $\tilde{s}_{1 \theta}$. On the other hand, we readily check, by contradiction, that the sets $\tilde{\mathcal{T}}_{i \bar{\epsilon}_{i}}$ are positively invariant (i.e. a trajectory in $\tilde{\mathcal{T}}_{i \bar{\epsilon}_{i}}$ remains in $\tilde{\mathcal{T}}_{i \bar{\epsilon}_{i}}$ for all the future time). Arguing as in the proof of Theorem 1, we generalize this result to the overall system. In other words, we have proved the following result:

Theorem 2. If the conditions $s_{i}>0$ are satisfied, for all $i \in I_{n}$, then the states ${ }_{195} x_{i t}$ of the perturbed system (1), where $\epsilon_{i}(t) \in\left(0, \bar{\epsilon}_{i}\right]$, for all $t \geq 0$, converge exponentially to the sets $\tilde{\mathcal{T}}_{i \bar{\epsilon}_{i}}$, where $0<\psi_{i}^{\dagger}<1$, for all $i \in I_{n}$.

\section{Stability analysis of the strictly positive steady state}

A strictly positive equilibrium $X^{e}$ reflects the surviving of all the generations of blood cells, which is the aim of a healthy hematopoiesis. When the condition (3) is satisfied, a unique $X^{e}$ exists. In this section, we answer one important open question about the problem of finding an estimate of the basin of attraction of $X^{e}$. Let us start by looking to the reintroduction functions, $\beta_{i}$ 's, from the resting to the proliferating stages. Owing to some biological considerations, Hill functions were proposed by Mackey in [12] to model $\beta_{i}$. Therefore, we consider,

$$
\beta_{i}\left(x_{i}\right)=\frac{\beta_{i}(0)}{1+b_{i} x_{i}^{\mathfrak{n}_{i}}},
$$

where $\beta_{i}(0)>0, b_{i}>0$ and $\mathfrak{n}_{i} \geq 2$. This typical choice was assumed in subsequent works ([1] and [16]). Actually, many other smoothly decreasing

functions $\beta_{i}$, with a finite maximum $\beta_{i}(0)$ and adjustable slope and inflection point, can be chosen to match the biological assumptions [12].

Throughout this section, we consider the functions $\beta_{i}$ in the form $(23)$ and we indicate later for which other forms our results remain valid. Since we are 
interested in the positive equilibrium $X^{e}$, we perform the change of coordinates, $\hat{x}_{i}=x_{i}-x_{i}^{e}$, for $i \in I_{n}$. It follows from (1) that

$$
\begin{aligned}
\dot{\hat{x}}_{i}(t)= & -\delta_{i}\left[\hat{x}_{i}(t)+x_{i}^{e}\right]-w_{i}\left(\hat{x}_{i}(t)+x_{i}^{e}\right) \\
& +2 L_{i} \int_{t-\tau_{i}}^{t} g_{i}(t-a) w_{i}\left(\hat{x}_{i}(a)+x_{i}^{e}\right) d a \\
& +2 K_{i-1} \int_{t-\tau_{i-1}}^{t} g_{i-1}(t-a) w_{i-1}\left(\hat{x}_{i-1}(a)+x_{i-1}^{e}\right) d a .
\end{aligned}
$$

However, a better representation of (24) that eases the analysis of its origin, can be obtained. Indeed, observe that, with an abuse of notation, $w_{i}\left(\hat{x}_{i}+x_{i}^{e}\right)=$ $w_{i}\left(x_{i}^{e}\right)+\mu_{i} \hat{x}_{i}+R_{i}\left(\hat{x}_{i}\right)$, where,

$$
\mu_{i}=\beta_{i}\left(x_{i}^{e}\right)+\beta_{i}^{\prime}\left(x_{i}^{e}\right) x_{i}^{e}, \quad \text { and, } \quad R_{i}\left(\hat{x}_{i}\right)=\int_{x_{i}^{e}}^{x_{i}^{e}+\hat{x}_{i}}\left[\hat{x}_{i}+x_{i}^{e}-l\right] w_{i}^{(2)}(l) d l .
$$

Moreover, we denote $\beta_{i *}=\delta_{i}+\mu_{i}$. It follows that 24 is equivalent to

$$
\begin{aligned}
\dot{\hat{x}}_{i}(t)= & -\beta_{i *} \hat{x}_{i}(t)+2 L_{i} \mu_{i} \int_{t-\tau_{i}}^{t} g_{i}(t-a) \hat{x}_{i}(a) d a \\
& -R_{i}\left(\hat{x}_{i}(t)\right)+2 L_{i} \int_{t-\tau_{i}}^{t} g_{i}(t-a) R_{i}\left(\hat{x}_{i}(a)\right) d a \\
& +2 K_{i-1} \mu_{i-1} \int_{t-\tau_{i-1}}^{t} g_{i-1}(t-a) \hat{x}_{i-1}(a) d a \\
& +2 K_{i-1} \int_{t-\tau_{i-1}}^{t} g_{i-1}(t-a) R_{i-1}\left(\hat{x}_{i-1}(a)\right) d a .
\end{aligned}
$$

Remark 3. Compared with Section 3, the stability analysis of the origin of (26) is more complicated due to the shifting. Indeed, linear functionals can no longer be used since the system (26) is not positive. A common approach to investigate the stability properties of such a class of systems is by using quadratic functions or functionals, as illustrated in the sequel.

\subsection{Introductory result}

To get a first intuition, let us consider the subsystem $(26)$ for $i=1$. A linear approximation at its origin is obtained by neglecting the terms where $R_{1}$ is present. The following linear system is obtained:

$$
\dot{z}_{1}(t)=-\beta_{1 *} z_{1}(t)+2 L_{1} \mu_{1} \int_{t-\tau_{1}}^{t} g_{1}(t-a) z_{1}(a) d a .
$$

Let us consider the positive definite quadratic function

$$
Q(a)=\frac{1}{2} a^{2} .
$$


We apply the Razumikhin's theorem (see, for instance, [7): Pick $q>1$ and consider $t \geq 0$ such that $q Q\left(z_{1}(t)\right) \geq Q\left(z_{1}(a)\right), \quad \forall a \in\left(t-\tau_{1}, t\right)$. Then the derivative of $Q$ along the trajectories of the system (27) satisfies:

$$
\begin{aligned}
\dot{Q}(t) & \leq-2 \beta_{1 *} Q\left(z_{1}(t)\right)+4 \sqrt{Q\left(z_{1}(t)\right)} L_{1}\left|\mu_{1}\right| \int_{t-\tau_{1}}^{t} g_{1}(t-a) \sqrt{Q\left(z_{1}(a)\right)} d a \\
& \leq-2\left[\beta_{1 *}-2 \sqrt{q} L_{1}\left|\mu_{1}\right| C_{1}\right] Q\left(z_{1}(t)\right) .
\end{aligned}
$$

We conclude from Razumikhin's theorem that the condition $\beta_{1 *}-2 L_{1}\left|\mu_{1}\right| C_{1}>0$ is sufficient for the asymptotic stability of the origin of the system (27). This leads us to introduce, for all $i \in I_{n}$, the constants

$$
\varsigma_{i}=\beta_{i *}-2 L_{i}\left|\mu_{i}\right| C_{i}=\delta_{i}+\mu_{i}-2 L_{i}\left|\mu_{i}\right| C_{i},
$$

that will be of use later in the stability analysis of the nonlinear system, in the analytic expression of the quadratic Lyapunov-Krasovskii functionals and in the size of the region of attraction that we will provide.

\subsection{Estimate of the basin of attraction of the positive steady state}

Contrary to Section 3, the nonpositivity of the system under study motivates the use of the positive definite function (28), as well as the following functionals:

$$
\begin{gathered}
\Omega_{i}\left(\varphi_{i t}\right)=\int_{t-\tau_{i}}^{t} \int_{l}^{t} g_{i}\left(l-a+\tau_{i}\right) Q\left(\varphi_{i}(a)\right) d a d l, \\
\Lambda_{i}\left(\varphi_{i t}\right)=\int_{t-\tau_{i}}^{t} e^{l-t} \int_{l}^{t} g_{i}\left(l-a+\tau_{i}\right) Q\left(\varphi_{i}(a)\right) d a d l .
\end{gathered}
$$

Notice that other types of functionals may be used instead of (31) and (32). However, for the sake of clarity, we use a weighted combination of them in order to compensate the distributed delayed terms and estimate the exponential decay rates. Moreover, we define for all $i \in I_{n}$, the following functionals:

$$
\begin{gathered}
S_{i}\left(\hat{x}_{i t}\right)=\frac{1}{2} Q\left(\hat{x}_{i}(t)\right)+L_{i}\left|\mu_{i}\right| \Omega_{i}\left(\hat{x}_{i t}\right), \\
N_{1}\left(\hat{x}_{1 t}\right)=S_{1}\left(\hat{x}_{1 t}\right)+\frac{\varsigma_{1}}{2 C_{1}} \Lambda_{1}\left(\hat{x}_{1 t}\right), \text { and for all } i \in\{2, \ldots, n\}, \\
N_{i}\left(\hat{x}_{i t}, \hat{x}_{i-1 t}\right)=S_{i}\left(\hat{x}_{i t}\right)+\frac{\varsigma_{i}}{2 C_{i}} \Lambda_{i}\left(\hat{x}_{i t}\right)+\psi_{i} \Lambda_{i-1}\left(\hat{x}_{i-1 t}\right),
\end{gathered}
$$

with $\varsigma_{i}$ the constants defined in Section 4.1, and $\psi_{i}$ are appropriate strictly positive constants to be selected later, for all $i \in\{2, \ldots, n\}$.

Next, for a later use, we prove in Appendix A the following assertion:

Claim 1. There exist constants $\hat{s}_{i}>0$, for all $i \in I_{n}$, which depend on the biological parameters of the model and on the strictly positive equilibrium $X^{e}$, such that, for all $\hat{x}_{i}>-x_{i}^{e}$, where $x_{i}^{e}>0$, the following inequality holds true:

$$
\left|R_{i}\left(\hat{x}_{i}\right)\right| \leq \hat{s}_{i} Q\left(\hat{x}_{i}\right) \text {, where, } \hat{s}_{i}>0 \text { are given in Appendix } A \text {. }
$$


${ }_{215}$ Remark 4. It is worth mentioning that the stability analysis which will be performed for the origin of the nonlinear system (26) is available for many other reintroduction functions $\beta_{i}$, as long as they satisfy the sector conditions (36).

Furthermore, in order to ease the notation, we denote

$$
I_{i}\left(\hat{x}_{i t}\right)=\int_{t-\tau_{i}}^{t} g_{i}(t-a) Q\left(\hat{x}_{i}(a)\right) d a .
$$

Finally, we define the constants $\tilde{k}_{i}=\frac{\varsigma_{i}}{8 \hat{s}_{i}}, \hat{k}_{i}=\frac{\varsigma_{i}}{4 C_{i} L_{i} \hat{s}_{i} e^{\tau_{i}}}$ and $\bar{N}_{i}=\min \left\{\tilde{k}_{i}^{2}, \hat{k}_{i}^{2}\right\}$. Notice that for all $i \in I_{n}, \tilde{k}_{i}$ and $\hat{k}_{i}$ are only dependent on the constant biological

Theorem 3. Let the system (26) be such that

$$
\varsigma_{i}>0
$$

for all $i \in I_{n}$. Then all the solutions of 26 with initial conditions $\hat{\varphi}_{i} \in$ $\mathcal{C}\left(\left[-\tau_{i}, 0\right],\left(-x_{i}^{e},+\infty\right)\right)$ satisfying

$$
N_{i}\left(\hat{\varphi}_{i}, \hat{\varphi}_{i-1}\right)<\bar{N}_{i}
$$

converge exponentially to the origin.

Remark 5. Generally, Lyapunov theory provides sufficient conditions for stability. Nevertheless, due to earlier published works we can comment conditions (38). In previous works (using frequency domain approaches), it was claimed 225 in [1] that the origin is locally asymptotically stable if $\delta_{i}+\left(2 L_{i} C_{i}+1\right) \mu_{i}>0$ is satisfied. However, in [16], it was shown that the previous assertion holds true only when $-\delta_{i}<\mu_{i}<0$. We notice that our stability conditions (38) are equivalent to those of [16] on that interval. Next, when $\mu_{i}>0$, our exponential stability conditions (38) (which are provided without specifying a particular 230 form of $f_{i}$ ), correspond to the conditions for local stability provided in [16] (and which have been slightly improved using Nyquist criterion for a typical selection of the functions $f_{i}$ in [16]). It remains the case $\mu_{i}<-\delta_{i}$ which is not covered by the Lyapunov approach proposed here, and which was addressed in [16]. The region of attraction defined in (39) is rather difficult to interpret. In fact, based on some numerical simulations and the conjecture made in [16], we suggest that the region defined in (39) is conservative.

Proof. First, let us observe for later use that the derivatives of the functionals $\Omega_{i}$ and $\Lambda_{i}$, for all $i \in I_{n}$, along the trajectories of $(26)$ satisfy,

$$
\begin{gathered}
\dot{\Omega}_{i}(t)=C_{i} Q\left(\hat{x}_{i}(t)\right)-\int_{t-\tau_{i}}^{t} g_{i}(t-a) Q\left(\hat{x}_{i}(a)\right) d a, \text { and }, \\
\dot{\Lambda}_{i}(t) \leq-\Lambda_{i}\left(\hat{x}_{i t}\right)-e^{-\tau_{i}} \int_{t-\tau_{i}}^{t} g_{i}(t-a) Q\left(\hat{x}_{i}(a)\right) d a+C_{i} Q\left(\hat{x}_{i}(t)\right),
\end{gathered}
$$


where the last inequality is a consequence of (2). For the sake of clarity, we will decompose now the proof of Theorem 3 in two parts: we prove the exponential stability of solutions of the first compartment $(i=1)$, and then we extend the result to any number of compartments $(i \geq 1)$.

i) LKF for the first compartment: We start with the first generation of hematopoietic stem cells. Using (36), one can prove that the derivative of the function $Q\left(\hat{x}_{1}(t)\right)$, introduced in $(28)$, along the trajectories of 26 satisfies

$$
\begin{gathered}
\dot{Q}(t) \leq 2\left[-\beta_{1 *}+L_{1}\left|\mu_{1}\right| C_{1}\right] Q\left(\hat{x}_{1}(t)\right)+\hat{s}_{1}\left|\hat{x}_{1}(t)\right| Q\left(\hat{x}_{1}(t)\right) \\
+2 L_{1}\left(\left|\mu_{1}\right|+\hat{s}_{1}\left|\hat{x}_{1}(t)\right|\right) I_{1}\left(\hat{x}_{1 t}\right) .
\end{gathered}
$$

It follows that the derivative of the functional $N_{1}$, introduced in (34), satisfies

$$
\begin{aligned}
\dot{N}_{1}(t) \leq & -\left[\frac{\varsigma_{1}}{8} Q\left(\hat{x}_{1}(t)\right)+\frac{\varsigma_{1}}{2 C_{1}} \Lambda_{1}\left(\hat{x}_{1 t}\right)\right]+\left[\frac{\hat{s}_{1}}{2}\left|\hat{x}_{1}(t)\right|-\frac{\varsigma_{1}}{4}\right] Q\left(\hat{x}_{1}(t)\right) \\
& -\frac{\varsigma_{1}}{8} Q\left(\hat{x}_{1}(t)\right)+\left[L_{1} \hat{s}_{1}\left|\hat{x}_{1}(t)\right|-\frac{\varsigma_{1} e^{-\tau_{1}}}{2 C_{1}}\right] I_{1}\left(\hat{x}_{1 t}\right) .
\end{aligned}
$$

On the other hand, from the definition of $N_{1}$ we observe that

$$
N_{1}\left(\hat{x}_{1 t}\right) \leq \frac{1}{2} Q\left(\hat{x}_{1}(t)\right)+\left(\frac{\varsigma_{1}+2 L_{1} C_{1}\left|\mu_{1}\right| e^{\tau_{1}}}{2 C_{1}}\right) \Lambda_{1}\left(\hat{x}_{1 t}\right) .
$$

From 41 and 42 , we deduce that for all $\tilde{\varsigma}_{1} \in\left(0, \min \left\{\frac{\varsigma_{1}}{4}, \frac{\varsigma_{1}}{\varsigma_{1}+2 L_{1} C_{1}\left|\mu_{1}\right| e^{\tau_{1}}}\right\}\right)$, the derivative of the functional $N_{1}$ satisfies

$$
\begin{aligned}
\dot{N}_{1}(t) \leq & -\tilde{\varsigma}_{1} N_{1}\left(\hat{x}_{1 t}\right)+\left[\frac{\hat{s}_{1}}{2}\left|\hat{x}_{1}(t)\right|-\frac{\varsigma_{1}}{4}\right] Q_{1}\left(\hat{x}_{1}(t)\right) \\
& +\left[L_{1} \hat{s}_{1}\left|\hat{x}_{1}(t)\right|-\frac{\varsigma_{1} e^{-\tau_{1}}}{2 C_{1}}\right] I_{1}\left(\hat{x}_{1 t}\right)-\frac{\varsigma_{1}}{8} Q\left(\hat{x}_{1}(t)\right) .
\end{aligned}
$$

From the definition of $N_{1}$, which is given in 34 , we notice that $\left|\hat{x}_{1}(t)\right| \leq$ $2 \sqrt{N_{1}\left(\hat{x}_{1 t}\right)}$. A direct consequence is that

$$
\begin{aligned}
\dot{N}_{1}(t) \leq & \left.-\tilde{\varsigma}_{1} N_{1}\left(\hat{x}_{1 t}\right)+\left[\hat{s}_{1} \sqrt{N_{1}\left(\hat{x}_{1 t}\right.}\right)-\frac{\varsigma_{1}}{4}\right] Q_{1}\left(\hat{x}_{1}(t)\right) \\
& \left.+\left[2 L_{1} \hat{s}_{1} \sqrt{N_{1}\left(\hat{x}_{1 t}\right.}\right)-\frac{\varsigma_{1} e^{-\tau_{1}}}{2 C_{1}}\right] I_{1}\left(\hat{x}_{1 t}\right)-\frac{\varsigma_{1}}{8} Q\left(\hat{x}_{1}(t)\right) .
\end{aligned}
$$

Now, we conclude that if the condition 39 is satisfied, then

$$
\dot{N}_{1}(t) \leq-\tilde{\varsigma}_{1} N_{1}\left(\hat{x}_{1 t}\right)-\frac{\varsigma_{1}}{8} Q\left(\hat{x}_{1}(t)\right) \text {. }
$$

This allows us to conclude that the origin of the subsystem $(26)$, for $i=1$, is exponentially stable, with a decay rate smaller than $\tilde{\varsigma}_{1}$.

ii) LKF for the overall system: Here we take into account all generations of immature blood cells. We use the inequality $\left|\hat{x}_{i}(t) \hat{x}_{i-1}(a)\right| \leq \xi_{i} Q\left(\hat{x}_{i}(t)\right)+$ 
$\frac{1}{\xi_{i}} Q\left(\hat{x}_{i-1}(a)\right)$, with $\xi_{i}>0$ for all $i>1$, and the inequality $\left|\hat{x}_{i}(t) \hat{x}_{i}(a)\right| \leq$ $Q\left(\hat{x}_{i}(t)\right)+Q\left(\hat{x}_{i}(a)\right)$, for all $i \in I_{n}$, and we select $\psi_{i}=\frac{K_{i-1}\left|\mu_{i-1}\right| e^{\tau_{i-1}}}{\xi_{i}}+\frac{\varsigma_{i} e^{-\tau_{i}} K_{i-1} \hat{s}_{i-1}}{2 L_{i} \hat{s}_{i} C_{i}} e^{\tau_{i-1}}$. Then the derivatives of the functions $Q\left(\hat{x}_{i}(t)\right)$, for all $i>1$, along the trajectories of (26) satisfy

$$
\begin{aligned}
\dot{Q}(t) \leq & 2\left[-\beta_{i *}+L_{i}\left|\mu_{i}\right| C_{i}\right] Q\left(\hat{x}_{i}(t)\right)+\hat{s}_{i}\left|\hat{x}_{i}(t)\right| Q\left(\hat{x}_{i}(t)\right) \\
& +2 L_{i}\left(\left|\mu_{i}\right|+\hat{s}_{i}\left|\hat{x}_{i}(t)\right|\right) I_{i}\left(\hat{x}_{i t}\right)+2 K_{i-1}\left|\mu_{i-1}\right| C_{i-1} \xi_{i} Q\left(\hat{x}_{i}(t)\right) \\
& +2 K_{i-1}\left(\hat{s}_{i-1}\left|\hat{x}_{i}(t)\right|+\frac{\left|\mu_{i-1}\right|}{\xi_{i}}\right) I_{i-1}\left(\hat{x}_{i-1 t}\right) .
\end{aligned}
$$

Moreover, we choose $\xi_{i}=\frac{\varsigma_{i}}{4 K_{i-1}\left|\mu_{i-1}\right| C_{i-1}}$. It follows that

$$
\begin{aligned}
\dot{N}_{i}(t) \leq & -\tilde{\varsigma}_{i} N_{i}\left(\hat{x}_{i}, \hat{x}_{i-1}\right)+\psi_{i} C_{i-1} Q\left(\hat{x}_{i-1}(t)\right)-\left[\frac{\varsigma_{i}}{8}-\frac{1}{2} \hat{s}_{i}\left|\hat{x}_{i}(t)\right|\right] Q\left(\hat{x}_{i}(t)\right) \\
& -\frac{\varsigma_{i}}{16} Q\left(\hat{x}_{i}(t)\right)+L_{i} \hat{s}_{i}\left[\left|\hat{x}_{i}(t)\right|-\frac{\varsigma_{i} e^{-\tau_{i}}}{2 L_{i} \hat{s}_{i} C_{i}}\right] I_{i}\left(\hat{x}_{i t}\right) \\
& +K_{i-1} \hat{s}_{i-1}\left[\left|\hat{x}_{i}(t)\right|-\frac{\varsigma_{i} e^{-\tau_{i}}}{2 L_{i} \hat{s}_{i} C_{i}}\right] I_{i-1}\left(\hat{x}_{i-1 t}\right),
\end{aligned}
$$

with $\tilde{\varsigma_{i}}>0$. Finally, we conclude that if the conditions $(39)$ are satisfied, then

$$
\dot{N}_{i}(t) \leq-\tilde{\varsigma}_{i} N_{i}\left(\hat{x}_{i t}, \hat{x}_{i-1 t}\right)-\frac{\varsigma_{i}}{16} Q\left(\hat{x}_{i}(t)\right)+\psi_{i} C_{i-1} Q\left(\hat{x}_{i-1}(t)\right) .
$$

As we had done in [6], we can prove that the derivative of the functional $W\left(\hat{X}_{t}\right)=\sum_{i=1}^{n} p_{i} N_{i}\left(\hat{x}_{i t}, \hat{x}_{i-1 t}\right)$, with an abuse of notation for $N_{1}$, and where $p_{i}=2^{n-i} \prod_{k=i+1}^{n} \frac{8 \psi_{k} C_{k-1}}{\varsigma_{k-1}}, p_{n}=1$, and $\hat{X}=\left(\hat{x}_{1}, \ldots, \hat{x}_{n}\right)$, satisfies,

$\dot{W}(t) \leq-\sum_{i=1}^{n} p_{i} \tilde{\varsigma}_{i} N_{i}\left(\hat{x}_{i t}, \hat{x}_{i-1 t}\right)-\frac{\varsigma_{1}}{8} Q\left(\hat{x}_{1}(t)\right)-\frac{\varsigma_{n}}{16} Q\left(\hat{x}_{n}(t)\right)-\frac{1}{2} \sum_{i=1}^{n-1} \frac{p_{i} \varsigma_{i}}{8} Q\left(\hat{x}_{i}(t)\right)$.

Finally, we obtain $\dot{W}(t) \leq-\varsigma W\left(\hat{X}_{t}\right)$, with $\varsigma=\min \left\{\tilde{\varsigma}_{1}, \ldots, \tilde{\varsigma}_{n}\right\}>0$.

To summarize, by virtue of the properties of the functionals $N_{i}$, for all $i \in I_{n}$, and since the original system (1) is a positive system, we conclude that the set

$$
\mathcal{A}=\left\{\varphi_{i} \in \mathcal{C}\left(\left[-\tau_{i}, 0\right], \mathbb{R}^{+}\right): N_{i}\left(\varphi_{i}-x_{i}^{e}, \varphi_{i-1}-x_{i-1}^{e}\right)<\bar{N}_{i}\right\}
$$

is a subset of the basin of attraction of the positive steady state of system (1).

Example 3. In this numerical example, we consider the system with the following biological functions and parameters for $n=3$ :

\begin{tabular}{|c||c||c||c||c||c||c|}
\hline & $\beta_{i}\left(x_{i}\right)$ & $f_{i}(a)$ & $\delta_{i}$ & $\tau_{i}$ & $\gamma_{i}$ & $K_{i}$ \\
\hline$i=1$ & $\frac{0.5}{1+x_{1}^{2}}$ & $\frac{10 e^{10 a}}{e^{10 \tau}-1}$ & 0.1356 & 1.109402 & 0.3 & 0.05 \\
\hline$i=2$ & $\frac{1}{1+x_{2}^{4}}$ & $\frac{10 e^{10 a}}{e^{10 \tau_{2}-1}}$ & 0.1669 & 1.2 & 0.4 & 0.07 \\
\hline$i=3$ & $\frac{3}{1+x_{3}^{2}}$ & $\frac{2 e^{2 a}}{e^{2 \tau_{2}-1}}$ & 0.3559 & 1.36 & 0.45 & 0.085 \\
\hline
\end{tabular}


From the selected parameters, it follows that

250

\begin{tabular}{|c||c||c||c||c||c|}
\hline & $x_{i}^{e}$ & $\alpha_{i}$ & $\varsigma_{i}$ & $\hat{s}_{i}$ & $\bar{N}_{i}$ \\
\hline$i=1$ & 0.70036 & 0.40422 & 0.08924 & 0.65070 & $2.5935 \times 10^{-4}$ \\
\hline$i=2$ & 0.78225 & 0.19888 & 0.02329 & 3.00487 & $9.3935 \times 10^{-7}$ \\
\hline$i=3$ & 1.0050 & 0.20422 & 0.33938 & 2.98491 & $2.02 \times 10^{-4}$ \\
\hline
\end{tabular}

We select constant initial conditions: $\varphi_{1}=0.6850, \varphi_{2}=0.782$ and $\varphi_{3}=$ 0.979. Therefore, we get, $N_{1}\left(\varphi_{1}-x_{1}^{e}\right)=7.16 \times 10^{-5}<\bar{N}_{1}, N_{2}\left(\varphi_{2}-x_{2}^{e}, \varphi_{1}-\right.$ $\left.x_{1}^{e}\right)=6.65 \times 10^{-7}<\bar{N}_{2}$, and, $N_{3}\left(\varphi_{3}-x_{3}^{e}, \varphi_{2}-x_{2}^{e}\right)=1.94 \times 10^{-4}<\bar{N}_{3}$. According to Theorem 3 , the positive steady state $\left(x_{1}^{e}, x_{2}^{e}, x_{3}^{e}\right)$ is exponentially stable (Figure 4).

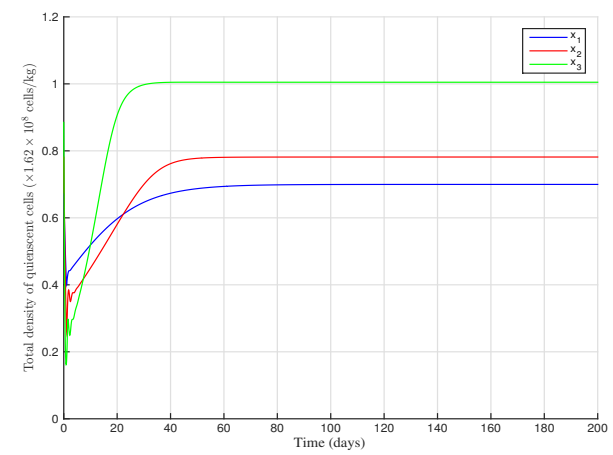

Figure 4: Trajectories $x_{1}, x_{2}$ and $x_{3}$ for the parameters of Example 3.

\subsection{Robustness analysis of the positive equilibrium point}

Here we return to the perturbed system (1), that we rewrite in a form similar to (26) by performing the change of coordinate $\hat{x}_{i}(t)=x_{i}(t)-x_{i}^{e}$. Based on the functionals constructed in Theorem 3, we prove the following result:

Corollary 2. Let the system (26) be perturbed by nonvanishing additive disturbances $\epsilon_{i}(t) \in\left(0, \epsilon_{i}\right], \bar{\epsilon}_{i}>0$, for all $t>0$, and $i \in I_{n}$. If the conditions

$$
\varsigma_{i}>0
$$

are satisfied for all $i \in I_{n}$, then all the solutions of 26] with initial conditions $\varphi_{i} \in \mathcal{C}\left(\left[-\tau_{i}, 0\right], \mathbb{R}^{+}\right)$satisfying

$$
\left(\frac{2 \bar{\epsilon}_{i}}{\theta \tilde{\varsigma}_{i}}\right)^{2} \leq N_{i}\left(\varphi_{i}-x_{i}^{e}, \varphi_{i-1}-x_{i-1}^{e}\right)<\bar{N}_{i}
$$

with $\theta \in(0,1)$, converge exponentially to the domain,

$$
\mathcal{G}_{\bar{\epsilon}_{i}}=\left\{\varphi_{i} \in \mathcal{C}\left(\left[-\tau_{i}, 0\right], \mathbb{R}^{+}\right), \quad N_{i}\left(\varphi_{i}-x_{i}^{e}, \varphi_{i-1}-x_{i-1}^{e}\right) \leq\left(\frac{2 \bar{\epsilon}_{i}}{\theta \tilde{\varsigma}_{i}}\right)^{2}\right\} .
$$


Proof. Let us prove the previous result for $i=1$. Arguing as we did in the proof of Theorem 3, one can generalize to the overall system. First, observe that the derivative of $Q\left(\hat{x}_{1}(t)\right)$ along the trajectories of the perturbed system satisfies:

$$
\begin{aligned}
\dot{Q}(t) \leq & 2\left[-\beta_{1 *}+L_{1}\left|\mu_{1}\right| C_{1}\right] Q\left(\hat{x}_{1}(t)\right)+\hat{s}_{1}\left|\hat{x}_{1}(t)\right| Q\left(\hat{x}_{1}(t)\right) \\
& +2 L_{1}\left(\left|\mu_{1}\right|+\hat{s}_{1}\left|\hat{x}_{1}(t)\right|\right) I_{1}\left(\hat{x}_{1 t}\right)+\left|\hat{x}_{1}(t)\right| \bar{\epsilon}_{i} .
\end{aligned}
$$

Consequently, the derivative of the functional $N_{1}$, introduced in (34), along the trajectories of the perturbed system, verifies

$$
\begin{aligned}
\dot{N}_{1}(t) \leq & -\left[\frac{\varsigma_{1}}{8} Q\left(\hat{x}_{1}(t)\right)+\frac{\varsigma_{1}}{2 C_{1}} \Lambda_{1}\left(\hat{x}_{1 t}\right)\right]+\left[\frac{\hat{s}_{1}}{2}\left|\hat{x}_{1}(t)\right|-\frac{\varsigma_{1}}{4}\right] Q\left(\hat{x}_{1}(t)\right) \\
& -\frac{\varsigma_{1}}{8} Q\left(\hat{x}_{1}(t)\right)+\left[L_{1} \hat{s}_{1}\left|\hat{x}_{1}(t)\right|-\frac{\varsigma_{1} e^{-\tau_{1}}}{2 C_{1}}\right] I_{1}\left(\hat{x}_{1 t}\right)+\left|\hat{x}_{1}(t)\right| \bar{\epsilon}_{i} .
\end{aligned}
$$

Using [42), and the fact that $\left|\hat{x}_{1}(t)\right| \leq 2 \sqrt{N_{1}\left(\hat{x}_{1 t}\right)}$, we obtain

$$
\begin{aligned}
\dot{N}_{1}(t) \leq & \left.-\tilde{\varsigma}_{1} N_{1}\left(\hat{x}_{1 t}\right)+\left[\hat{s}_{1} \sqrt{N_{1}\left(\hat{x}_{1 t}\right.}\right)-\frac{\varsigma_{1}}{4}\right] Q_{1}\left(\hat{x}_{1}(t)\right) \\
& +\left[2 L_{1} \hat{s}_{1} \sqrt{N_{1}\left(\hat{x}_{1 t}\right)}-\frac{\varsigma_{1} e^{-\tau_{1}}}{2 C_{1}}\right] I_{1}\left(\hat{x}_{1 t}\right)-\frac{\varsigma_{1}}{8} Q\left(\hat{x}_{1}(t)\right)+2 \bar{\epsilon}_{i} \sqrt{N_{1}\left(\hat{x}_{1 t}\right)},
\end{aligned}
$$

where $\tilde{\varsigma}_{1} \in\left(0, \min \left\{\frac{\varsigma_{1}}{4}, \frac{\varsigma_{1}}{\varsigma_{1}+2 L_{1} C_{1}\left|\mu_{1}\right| e^{\tau_{1}}}\right\}\right)$. Therefore, when $N_{1}\left(\varphi_{1}-x_{1}^{e}\right)<\bar{N}_{1}$ is satisfied, we deduce that

$$
\dot{N}_{1}(t) \leq-\tilde{\varsigma}_{1} N_{1}\left(\hat{x}_{1 t}\right)-\frac{\varsigma_{1}}{8} Q\left(\hat{x}_{1}(t)\right)+2 \bar{\epsilon}_{i} \sqrt{N_{1}\left(\hat{x}_{1 t}\right)} .
$$

Now, let us consider any $\theta \in(0,1)$ and observe that for all initial conditions $\varphi_{1}$ satisfying $N_{1}\left(\varphi_{1}-x_{1}^{e}\right)<\bar{N}_{1}$ with $\varphi_{1} \notin \mathcal{G}_{\bar{\epsilon}_{i}}$, the inequality (53) gives

$$
\dot{N}_{1}(t) \leq-(1-\theta) \tilde{\varsigma}_{1} N_{1}\left(\hat{x}_{1 t}\right) \text {. }
$$

We conclude that the states $x_{1 t}$ satisfying 49 converge exponentially to the invariant set $\mathcal{G}_{\bar{\epsilon}_{1}}$, defined in $(50)$, such that the decay rate of the trajectory $x_{1}(t)$ is smaller than, or equal to, $\frac{(1-\theta) \tilde{\varsigma}_{1}}{2}$.

\section{Conclusion}

With the aim of constantly refining and improving the modeling and the analysis of hematopoietic mechanisms, we proposed explicit constructions of suitable strict LKFs for nonlinear hematopoietic systems with distributed delays. Our approach allowed us to solve some practical and technical issues, which complement already published results on the topic. In comparison with previous works, our results provided exponential stability with an estimate on the decay rate of the solutions, and are derived without any extra assumption on the mitosis functions. Moreover, a robustness analysis was performed under 
nonvanishing perturbations (that may represent dedifferentiation flux, together with model uncertainties), and we have also covered some practical situations such that time-varying differentiating rates (to model the action on the blockade in differentiation and re-differentiation). Particular emphasis was given to the positive steady state that represents healthy hematopoiesis, and for which we provided an explicit formulation of a subset of its region of attraction. Future work will enhance the role of dedifferentiation and transdifferentiation by

considering hematopoietic models where cell plasticity is no more a marginal

280 phenomenon, and cannot be considered as a perturbation, but has to be fully modeled.

\section{References}

[1] M. Adimy, F. Crauste, A. Abdllaoui, Discrete Maturity-Structured Model of Cells Differentiation with Applications to Acute Myelogenous Leukemia, J. Biological Systems, No. 3, pp. 395-424, (2008).

[2] M. Adimy, F. Crauste, Mathematical Model of Hematopoiesis Dynamics with Growth Factor-dependent Apoptosis and Proliferation regulations, Math. Comput. Modelling 49, No. 11-12, pp. 2128-2137, (2009).

[3] M. Adimy, F. Crauste, A. Abdllaoui, Boundedness and Lyapunov function for a nonlinear system of hematopoietic stem cell dynamics, C. R. Acad. Sci. Paris, Ser. I, 348, No. 7-8, pp. 373-377, (2010).

[4] S. Cai, X. Fu, Z. Sheng, Dedifferentiation: a new approach in stem cell research, Bioscience 57.8, pp. 655-662, (2007).

[5] W. Djema, F. Mazenc, C. Bonnet, Lyapunov Stability Analysis of a Model Describing Hematopoiesis. Proceedings of the European Control Conference (ECC), Linz, Austria, pp. 2711-2716, (2015).

[6] W. Djema, F. Mazenc, C. Bonnet, Stability of Immature Cell Dynamics in Healthy and Unhealthy Hematopoiesis. Proceedings of the American Control Conference (ACC), Boston, USA, pp. 6121-6126, (2016).

[7] E. Fridman, Introduction to time-delay systems: analysis and control, Birkhauser, Systems and Control: Foundations and Applications, Springer, (2014).

[8] E. Fridman, C. Bonnet, F. Mazenc, W. Djema, Stability of the cell dynamics in Acute Myeloid Leukemia. Systems \& Control Letters Vol. 88, pp. 91-100, (2016).

[9] H. Khalil, Nonlinear systems, Third Edition. Prentice Hall, Upper Saddle River, NJ, (2002).

[10] V. Kolmanovskii, A. Myshkis, Introduction to the Theory and Applications of Functional Differential Equations, Kluwer Academic Publishers, Dordrecht, (1999). 
[11] E. Lainey, et al., Tyrosine kinase inhibitors for the treatment of acute myeloid leukemia: Delineation of anti-leukemic mechanisms of action, Biochemical Pharmacology 82, pp. 1457-1466, (2011).

[12] M.C. Mackey, Unified hypothesis of the origin of aplastic anemia and periodic hematopoiesis, Blood, 51: pp. 941-956, (1978).

[13] J. M. Mahaffy, J. Bélair, M.C. Mackey, Hematopoietic Model with Moving Boundary Condition and State-delay: Applications in Erythropoiesis, Journal of Theoretical Biology, 190. No. 2, pp. 135-146, (1998).

[14] M. Malisoff, F. Mazenc, Constructions of Strict Lyapunov Functions, Serie : Communications and Control Engineering. Spinger-Verlag London Ltd, U.K., (2009).

[15] D. B. Sykes, et al. Inhibition of dihydroorotate dehydrogenase overcomes differentiation blockade in acute myeloid leukemia, Cell, 167.1, pp. 171-186, (2016).

325 [16] H. Özbay, C. Bonnet, H. Benjelloun, J. Clairambault, Stability analysis of cell dynamics in leukemia. Math. Model Nat. Phenom., Vol. 7, No. 1, pp. 203-234, (2012).

[17] H. Özbay, C. Bonnet, J. Clairambault, Stability analysis of systems with distributed delays and application to hematopoietic cell maturation dynamics. Conference on Decision and Control, Mexico, pp. 2050-2055, (2008).

[18] L. Pujo-Menjouet, S. Bernard, M.C. Mackey, Long period oscillations in a $G_{0}$ model of hematopoietic stem cells, SIAM. J. Appl. Dynam. Syst. 4(2): pp. 312-332, (2005).

\section{Appendix A. Proof of Claim 1}

For notational convenience, we drop the subscript " $i$ " and we use $x_{e}$ instead of $x_{i}^{e}$ to denote the positive equilibrium. Using the expression of $\beta$ given in 233, we observe that for all $x_{e}>0$ and $\mathfrak{z}>-x_{e}, R(\mathfrak{z})=\beta(0) J(\mathfrak{z})-\mu \mathfrak{z}$, where $J(\mathfrak{z})=\frac{\mathfrak{z}+x^{e}}{1+b\left(\mathfrak{z}+x_{e}\right)^{\mathfrak{n}}}-\frac{x_{e}}{1+b x_{e}^{\mathfrak{n}}}$. First, let us study the function

$$
\rho(\mathfrak{z})=\frac{1}{1+b\left(\mathfrak{z}+x_{e}\right)^{\mathfrak{n}}}-\frac{1}{1+b x_{e}^{\mathfrak{n}}}=\frac{b\left[x_{e}^{\mathfrak{n}}-\left(\mathfrak{z}+x_{e}\right)^{\mathfrak{n}}\right]}{p(\mathfrak{z})},
$$

where $p(\mathfrak{z})=\left[1+b\left(\mathfrak{z}+x_{e}\right)^{\mathfrak{n}}\right]\left(1+b x_{e}^{\mathfrak{n}}\right)$. Observe that

$$
\left(\mathfrak{z}+x_{e}\right)^{\mathfrak{n}}-x_{e}^{\mathfrak{n}}=\mathfrak{n} x_{e}^{\mathfrak{n}-1} \mathfrak{z}+\mathfrak{n} \int_{0}^{\mathfrak{z}} \int_{x_{e}}^{x_{e}+l}(\mathfrak{n}-1) m^{n-2} d m d l .
$$

335 Consequently, $\rho(\mathfrak{z})=-\frac{\mathfrak{n} b x_{e}^{\mathfrak{n}-1}}{p(\mathfrak{z})} \mathfrak{z}+\mathfrak{C}(\mathfrak{z})$, where, $\mathfrak{C}(\mathfrak{z})=-\frac{\mathfrak{n} b(\mathfrak{n}-1)}{p(\mathfrak{z})} \int_{0}^{\mathfrak{z}} \int_{0}^{l}\left(m+x_{e}\right)^{\mathfrak{n}-2} d m d l$. 
Denote $h=1+b x_{e}^{\mathfrak{n}}$ and observe that, $\frac{1}{p(\mathfrak{z})}=\frac{1}{h}\left(\rho(\mathfrak{z})+\frac{1}{h}\right)$. It follows that $\rho(\mathfrak{z})=-\mathfrak{n} b x_{e}^{\mathfrak{n}-1}\left(\frac{\rho(\mathfrak{z})}{h}+\frac{1}{h^{2}}\right) \mathfrak{z}+\mathfrak{C}(\mathfrak{z})$.

Consequently, we get the intermediate result:

$$
\rho(\mathfrak{z})=-\frac{\mathfrak{n} b x_{e}^{\mathfrak{n}-1}}{h^{2}} \mathfrak{z}+\mathfrak{C}(\mathfrak{z})-\frac{\mathfrak{n} b x_{e}^{\mathfrak{n}-1}}{h} \rho(\mathfrak{z}) \mathfrak{z} .
$$

On the other hand, observe that

$$
J(\mathfrak{z})=\left(\rho(\mathfrak{z})+\frac{1}{h}\right) \mathfrak{z}+x_{e} \rho(\mathfrak{z})=\mathfrak{c}_{1} \mathfrak{z}+\mathfrak{c}_{2} \mathfrak{C}(\mathfrak{z})+\mathfrak{c}_{3} \rho(\mathfrak{z}) \mathfrak{z},
$$

where the last equality is a direct consequence of A.1, with $\mathfrak{c}_{1}=\frac{1}{h}-\frac{\mathfrak{n} b x_{e}^{\mathfrak{n}}}{h^{2}}$, $\mathfrak{c}_{2}=x_{e}$ and $\mathfrak{c}_{3}=\left(1-\frac{\mathfrak{n} b x_{e}^{\mathfrak{n}}}{h}\right)$. Now, we readily check that

$$
|\mathfrak{C}(\mathfrak{z})| \leq \frac{\mathfrak{n} b(\mathfrak{n}-1)}{p(\mathfrak{z})}\left(|\mathfrak{z}|+x_{e}\right)^{\mathfrak{n}-2} \frac{\mathfrak{z}^{2}}{2} .
$$

It follows that $|\rho(\mathfrak{z})| \leq \frac{\mathfrak{n} b x_{e}^{\mathfrak{n}-1}}{p(\mathfrak{z})}|\mathfrak{z}|+|\mathfrak{C}(\mathfrak{z})|$. Using A.3, we get

$$
|\mathfrak{z} \rho(\mathfrak{z})| \leq \frac{\mathfrak{n} b x_{e}^{\mathfrak{n}-1}}{p(\mathfrak{z})} \mathfrak{z}^{2}+\frac{\mathfrak{n} b(\mathfrak{n}-1)}{2 p(\mathfrak{z})}\left(|\mathfrak{z}|+x_{e}\right)^{\mathfrak{n}-2}|\mathfrak{z}|^{3} .
$$

From A.2 , we deduce that,

$$
\begin{aligned}
\left|J(\mathfrak{z})-\mathfrak{c}_{1} \mathfrak{z}\right| \leq & \frac{\mathfrak{n} b(\mathfrak{n}-1)\left|\mathfrak{c}_{3}\right|}{2 p(\mathfrak{z})}\left(|\mathfrak{z}|+x_{e}\right)^{\mathfrak{n}-2}|\mathfrak{z}|^{3} \\
& +\left[\frac{\mathfrak{n} b(\mathfrak{n}-1)\left|\mathfrak{c}_{2}\right|\left(|\mathfrak{z}|+x_{e}\right)^{\mathfrak{n}-2}}{2 p(\mathfrak{z})}+\frac{\mathfrak{n} b x_{e}^{\mathfrak{n}-1}\left|\mathfrak{c}_{3}\right|}{p(\mathfrak{z})}\right] \mathfrak{z}^{2} .
\end{aligned}
$$

Now, observe that $\frac{1}{p(\mathfrak{z})}=\frac{1}{\left[1+b\left(\mathfrak{z}+x_{e}\right)^{\mathfrak{n}}\right] h}$. Therefore, when $\mathfrak{z} \geq 0$, we have

$$
\frac{1}{p(\mathfrak{z})}=\frac{1}{\left[1+b\left(|\mathfrak{z}|+x_{e}\right)^{\mathfrak{n}}\right] h}
$$

and when $\mathfrak{z} \leq 0$ ( i.e. $\left.\mathfrak{z} \in\left(-x_{e}, 0\right]\right)$, we get

$$
\frac{1}{p(\mathfrak{z})} \leq \frac{1}{h} \leq \frac{1+b\left(2 x_{e}\right)^{\mathfrak{n}}}{\left[1+b\left(|\mathfrak{z}|+x_{e}\right)^{\mathfrak{n}}\right] h} .
$$

Consequently, for all $\mathfrak{z}>-x_{e}$, we have

$$
\frac{1}{p(\mathfrak{z})} \leq \frac{1+b\left(2 x_{e}\right)^{\mathfrak{n}}}{\left[1+b\left(|\mathfrak{z}|+x_{e}\right)^{\mathfrak{n}}\right] h}
$$


We deduce that

$$
\begin{aligned}
\mid J(\mathfrak{z}) & -\mathfrak{c}_{1} \mathfrak{z} \mid \leq\left[\left(\frac{\mathfrak{n} b(\mathfrak{n}-1)\left|\mathfrak{c}_{3}\right|\left(1+b\left(2 x_{e}\right)^{\mathfrak{n}}\right)}{2 h}\right) \frac{\left(|\mathfrak{z}|+x_{e}\right)^{\mathfrak{n}-2}|\mathfrak{z}|}{1+b\left(|\mathfrak{z}|+x_{e}\right)^{\mathfrak{n}}}\right. \\
& \left.+\frac{\mathfrak{n} b(\mathfrak{n}-1)\left|\mathfrak{c}_{2}\right|\left(|\mathfrak{z}|+x_{e}\right)^{\mathfrak{n}-2}\left(1+b\left(2 x_{e}\right)^{\mathfrak{n}}\right)}{2\left[1+b\left(|\mathfrak{z}|+x_{e}\right)^{\mathfrak{n}}\right] h}+\frac{\mathfrak{n} b x_{e}^{\mathfrak{n}-1}\left|\mathfrak{c}_{3}\right|\left(1+b\left(2 x_{e}\right)^{\mathfrak{n}}\right)}{\left[1+b\left(|\mathfrak{z}|+x_{e}\right)^{\mathfrak{n}}\right] h}\right] \mathfrak{z}^{2} .
\end{aligned}
$$

By distinguishing between the two cases $|\mathfrak{z}|+x_{e} \geq 1$ and $|\mathfrak{z}|+x_{e} \leq 1$, one can prove that the following inequalities are satisfied for all $\mathfrak{z}>-x_{e}$,

$$
\frac{\left(|\mathfrak{z}|+x_{e}\right)^{\mathfrak{n}-2}|\mathfrak{z}|}{1+b\left(|\mathfrak{z}|+x_{e}\right)^{\mathfrak{n}}} \leq \frac{\left(|\mathfrak{z}|+x_{e}\right)^{\mathfrak{n}-1}}{1+b\left(|\mathfrak{z}|+x_{e}\right)^{\mathfrak{n}}} \leq \max \left\{b, b^{-1}\right\}
$$

It follows that $\left|J(\mathfrak{z})-\mathfrak{c}_{1} \mathfrak{z}\right| \leq \mathfrak{c}_{4} \mathfrak{z}^{2}$, with the positive constant $\mathfrak{c}_{4}=\frac{\mathfrak{n} b(\mathfrak{n}-1)\left(1+b\left(2 x_{e}\right)^{\mathfrak{n}}\right)\left(x_{e}+\left|\mathfrak{c}_{3}\right|\right) \max \left\{b, b^{-1}\right\}}{2 h}+\frac{\mathfrak{n} b x_{e}^{\mathfrak{n}-1}\left(1+b\left(2 x_{e}\right)^{\mathfrak{n}}\right)\left|\mathfrak{c}_{3}\right|}{h^{2}}$

On the other hand, we easily check that $\mu=\beta(0) \mathfrak{c}_{1}$, with $\mu$ defined in 25$]$. It follows that $|R(\mathfrak{z})| \leq \beta(0) \mathfrak{c}_{4} \mathfrak{z}^{2}$. Since $Q(\mathfrak{z})=\frac{1}{2} \mathfrak{z}^{2}$, we conclude that

$$
|R(\mathfrak{z})| \leq \hat{s} Q(\mathfrak{z}), \text { where, } \hat{s}=2 \mathfrak{c}_{4} \beta(0) .
$$

